\title{
YEAST GROWTH INFLUENCED BY PARALLEL COMBINATION OF TIME-VARYING AND STATIC LF EMF
}

Presented article deals with the mechanisms of low frequency electromagnetic field impact on biological structures. Special attention is paid to the theories describing the influence of applied electromagnetic field on microtubule structures and ions bound on the cell membrane surface. These theories are confronted with the experiments presented herein. For better reliability of the experiments, new exposure coil system has been designed and created. Using this exposure system, results are conducted on the eukaryotic cells represented by saccharomyces cerevisiae. Observed effects show a good correlation mainly with ion parametric resonance model predictions proposed by Lednev and are discussed in the scope of further investigation possibilities.

Keywords: Low frequency electromagnetic field, ion parametric resonance, magnetic flux density, saccharomyces cerevisiae.

\section{Introduction}

Investigations about non-thermal effect of low frequency electromagnetic field (LF EMF) applied on biological structures are still full of ambiguity and uncertainty. This is caused by the absence of a general theory about mechanisms on the cellular level that could be accepted by the whole scientific society. However, the research in this area has succeeded at least partially, because several theories have been established and published.

In this paper the two of the theories that seem to be most prominent in the research during last few years are briefly presented. Both of the theories are confronted with our experimental work, where the influence of LF EMF on the yeast strand VIVO is investigated. Findings and results are discussed within the conception of the mentioned theories, while some conclusions and recommendations for further work are presented.

\section{Interaction mechanizms between LF EMF and biological structures}

As it has been published in many studies before [1,2], the EMF is naturally presented (at least in the form of transmembrane potential) in biological environment and aligned with many processes (i.e. communication between cells, proliferation processes and others). But most recent scientific attention is paid to phenomenon of non-thermal connection between biosystems (albeit unicellular in our case) and man-made electromagnetic fields, at least in the specific frequency range. The investigations in this field showed a multitude of possibilities to pursue in the process of quan- tifying and elucidating the mechanisms of actions observed over the course of considered experiments. However, for the purpose of this work, two mechanisms that are currently probably most prominent have been chosen.

\section{A. Frohlich's theory of coherent oscilations}

The first mechanism is based on notions by H. Frohlich [3] and explores highly polar structures within living cells and vibrations thereof, creating conditions for endogenous electromagnetic field generation. This notion was further explored and developed by authors at the Institute of Photonics, Prague, Czech Republic and was also used to quantify the mechanism of non-invasive cancer detection proposed by Vedruccio et al. and since commercially manufactured under the name TrimProbe [4]. The basis of this theory lies in the presence of microtubules, hollow cylinders made of highly polar protein pairs ( $\alpha$ - and $\beta$-tubulin), Fig. 1.

Interestingly, these are most prominent during the division process, wherein they serve both as structural elements and locomotive helpers especially during the separation of nuclei of mother and daughter cells. Furthermore, experiments conducted by Pokorny et al. [5] showed significantly enhanced levels of electromagnetic activity during the budding phase of yeast cells and within synchronized yeast cells. Based on this theory it could be assumed that application of exogenous electromagnetic field within the frequency range employed by microtubules might lead to disruption of the underlying physiological function(s).

\section{B. Lednev's ion resonance theories}

The second mechanism lies in the interpretation of Lednev's Ion Parametric Resonance (IPR) model [6]. This model is based

\footnotetext{
* Roman Radil, Ladislav Janousek

Department of Electromagnetic and Biomedical Engineering, Faculty of Electrical Engineering, University of Zilina, Slovakia,

E-mail: roman.radil@fel.uniza.sk
} 


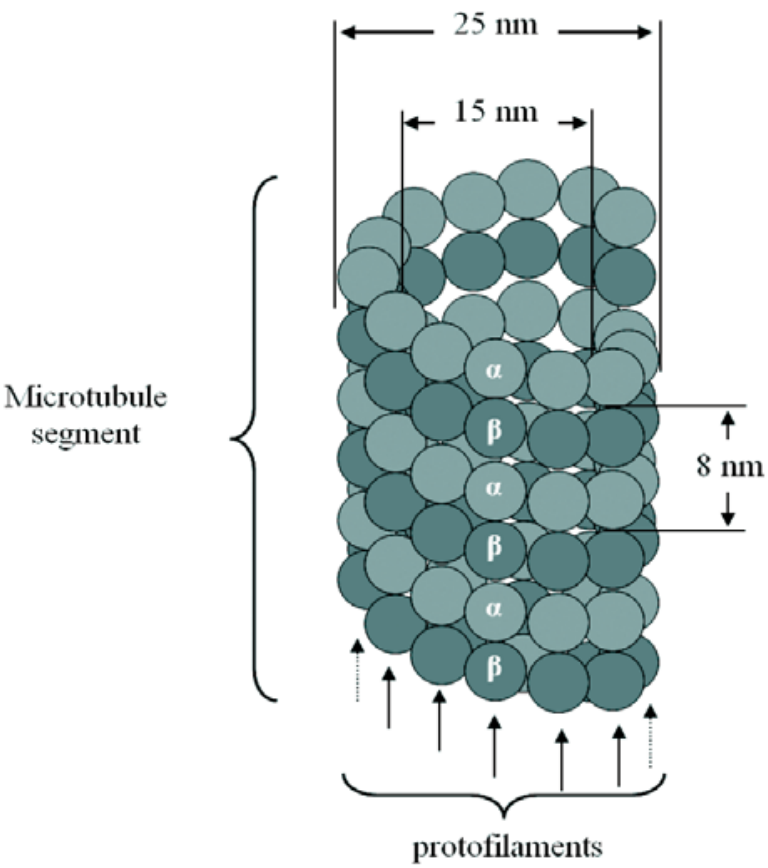

Fig. 1 Microtubule segment

5. Degenerated vibrational level of the ion with its characteristic angular frequency $\omega$ is split into three sublevels (Zeeman effect)

6. Oscillations of magnetic sublevels $\omega_{E 1}$ and $\omega_{E 2}$ are coherent amplitude and phase ratio is independent of time. The coherency allows interference interactions between the $\mathrm{Ca}^{2+}$ oscillator sublevels, which causes the biological response above the thermal noise level.

7. Oscillations are present only while the $\mathrm{Ca}^{2+}$ ion is bound to the specific protein location.

Based on these suggestions, Lednev in his works developed a model that could help with deduction of probability of specific ion transition to an energetically lower level, caused by application of external non-stationary magnetic field. This model was criticized by Adair [8] and defended by Engstrom [9]. The objections made by Adair were due to the spherical symmetry to enable the interference, and the lifetime of the excited ion state that must be of the order of Larmor precession; otherwise, the precession movement would be interrupted and the biological effect could not occur. Lednev addressed these objections by generalizing his theory to the case of continuous excitation and created a new model in 1996 [10], which led to some changes in assumptions from the previous model:

1. The ion displacement to the binding location on the protein is considered as the beginning of the excitation.

2. Magnetic fields (static, time-varying, combined) invoke precession of vibrational axes of $\mathrm{Ca}^{2+}$ oscillator depending on the orientation of the magnetic field. In certain combination of relaxing time of $\mathrm{Ca}^{2+}$ oscillator and applied field parameters, there is a possibility to achieve a considerable change of polarization level in a plane perpendicular to the applied magnetic field.

applied to a biosystem, first described by McLeod and Liboff in [7]. The model is principally shown in Fig. 2 and can be interpreted as an adaptation of atomic spectroscopy to biological environment.

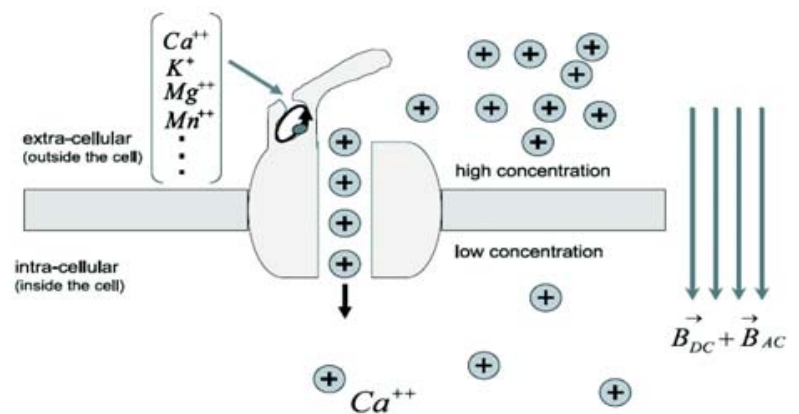

Fig. 2 Bound-ion polarization caused by a combination of static and time-varying magnetic field

The first version (1991) emanates from following assumptions:

1. The biosystem reaction onto the applied exogenous field is based on presence of $\mathrm{Ca}^{2+}$ ion bound to a specific protein location, enzymatic activity of which, is under control of such ion.

2. The bound ion is considered as isotropic, charged oscillator.

3. Oscillations are excited by thermal changes in biosystem.

4. $\mathrm{Ca}^{2+}$ ion's potential path is of spherical symmetry.

3. It is assumed that the probability of structure modification in $\mathrm{Ca}^{2+}$ bound location depends on time-average level of polarization of $\mathrm{Ca}^{2+}$ oscillator.

The resulting probability of energy density is given by:

$$
p=\frac{\Psi x^{2}-\Psi y^{2}}{\Psi x^{2}+\Psi y^{2}}
$$

where $\Psi$ represents the electromagnetic field intensity in the direction of the given axis.

This model was proposed on the basis of the experimental findings [8], where the magnetic flux density was lower than it was in previous cases. Spin movement of a particle placed into the exogenous magnetic field is described by Larmor precession $\omega_{L}$ :

$$
\omega_{L}=\gamma B_{0},
$$

where $\gamma$ is the value of gyromagnetic ratio of a nucleus and $B_{0}$ is the magnetic flux density of the static magnetic (DC) field. Even in this case, the combination of time-varying and static magnetic field causes frequency modulation of the resulting electromagnetic field, intensity of which is:

$$
\Psi=\Psi_{0} \Sigma J_{n}\left(\gamma \frac{B_{1}}{f}\right) e^{i\left(\omega_{L}+2 \pi n f\right) t},
$$


where $J_{n}$ represents Bessel function of n-th order, $f$ is frequency, $B_{1}$ represents magnetic flux density of time-varying (AC) magnetic field, which causes the modulation and $t$ is the time. Statistically significant bio-effect was found during the application of fields in the $\mu \mathrm{T}$ range. Nowadays, this model is one of the most important subjects in many experimental investigations and its further development is highly probable.

\section{Exposure system}

For the purpose of our experiments, the incubator described in [11] was used. To obtain more exact results, it was decided that inhomogeneity should not exceed 5\% of the applied LF EMF. That's why a new exposure coil system has to be designed. The new system proposal is based on numerical modeling. The model consists of three air coils. The role of the upper and lower side coils is to generate the LF EMF field of desired strength (mean value of $2.4 \mathrm{mT}$ ) and to complement the centre coil which further enhances the homogeneity of the generated EMF (max. $5 \%$ variation within the exposed Petri dishes).

Number of turns for each of the side coils was determined to 105 , wound in 14 layers. The stabilization coil was designed with 56 turns, wound in 4 layers. The results of numerical simulations, presented in Fig. 3 showed that the desired homogeneity in the exposure area was achieved at the $1 \mathrm{~A}$ of excitation current of a sinusoidal shape. Minimum value of magnetic flux density in exposure area was $2.36 \mathrm{mT}$ and maximum $2.47 \mathrm{mT}$.

As it is demonstrated in Fig. 3, the proposed coil system is of uncommon shape, so the atypical support system for its creation has to be designed.

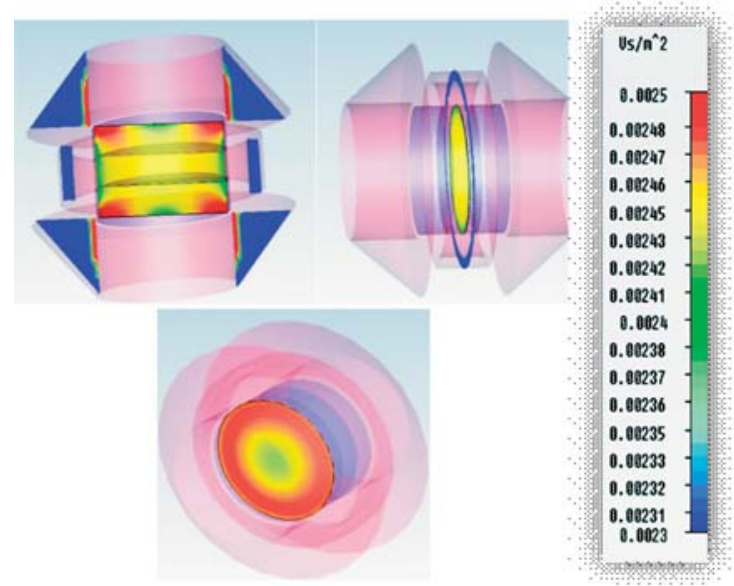

Fig. 3 Proposed model of exposure coil system-simulation results of $B$ field; color range from 2.3 to $2.5 \mathrm{mT}$

This task was solved in cooperation with the Faculty of Mechanical Engineering, University of Zilina. Model of the coil strut was designed in the Autodesk Inventor software, sent to a 3D printer and printed. The comparison of the model and output from the 3D printer can be seen in Fig. 4 .
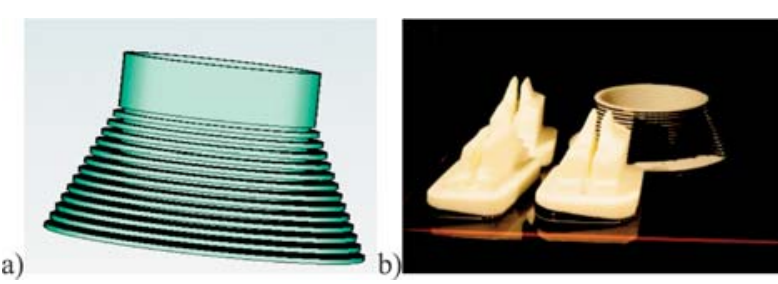

Fig. 4 a) Model of the coil strut b) Output from the 3D printer - the strut is in the background of the picture

The final creation of the proposed coil system was made manually using about $100 \mathrm{~m}$ of enameled copper winding wire with the diameter of $1.8 \mathrm{~mm}$. The three coils were formed separately and connected together in series as shown in Fig. 5.

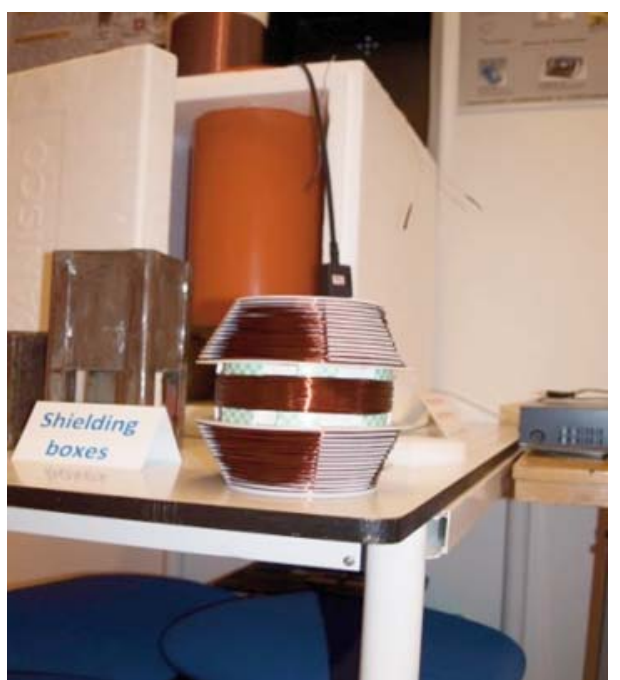

Fig. 5 The finalized exposure coil system in front of the incubator

Inductance of the whole coil system is $L=5.346 \mathrm{mH}$, measured by an LC-meter. The magnetic flux density(B)-field produced by the coil system was measured by flux-gate sensor at $0.1 \mathrm{~A}$ sinusoidal current and was recalculated to $1 \mathrm{~A}$ (because of linear dependence). The values from 2.37 to $2.49 \mathrm{mT}$ confirmed that the constructed coil system is adequate to the simulation proposal. Due to the similar B with the coil used in [11] (B was 2.0 - 2.3 mT) the results from experiments presented herein could be compared with the results from previous experiments made by the authors.

\section{Experimental work}

As it has been mentioned in previous section of this article, this experiment is focused on the behavior of saccharomyces cere- 
visiae affected by exogenous time-varying LF EMF produced by the newly designed coil system. At the beginning of the experiment - three petri dishes (exposed samples) are placed to the proper position in the coil and three are arranged into the magnetic shielding box and inserted to the incubator, which is principally shown in Fig. 6.

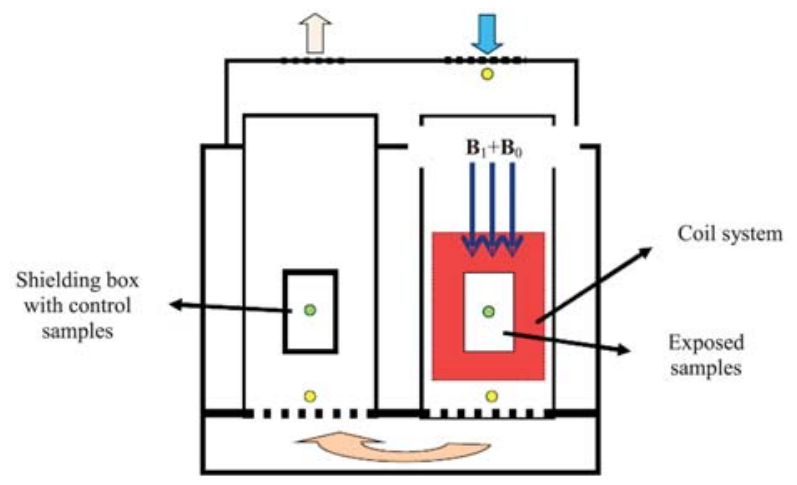

Fig. 6 Principal scheme of the incubator

Temperature of both exposed and unexposed samples is monitored by thermistors during the experiment. Frequency of applied field is $1.6 \mathrm{kHz}$ at the current of sinusoidal shape with amplitude $1.01 \mathrm{~A}$. The mentioned frequency was chosen to compare the results from [12], where statistically significant bio-effect occurred at this frequency.

Once the exposure system parameters are set up, the exposure has started. Time of the exposure is 66 hours according to $[12,13]$. After the exposure, the petri dishes are scanned and evaluated using the application Petri scanner - software for counting of yeast colonies and growth (surface) area evaluation.

The first experiment was built-up according to the IPR theory [10], so the B vector of AC field produced by the coil system is

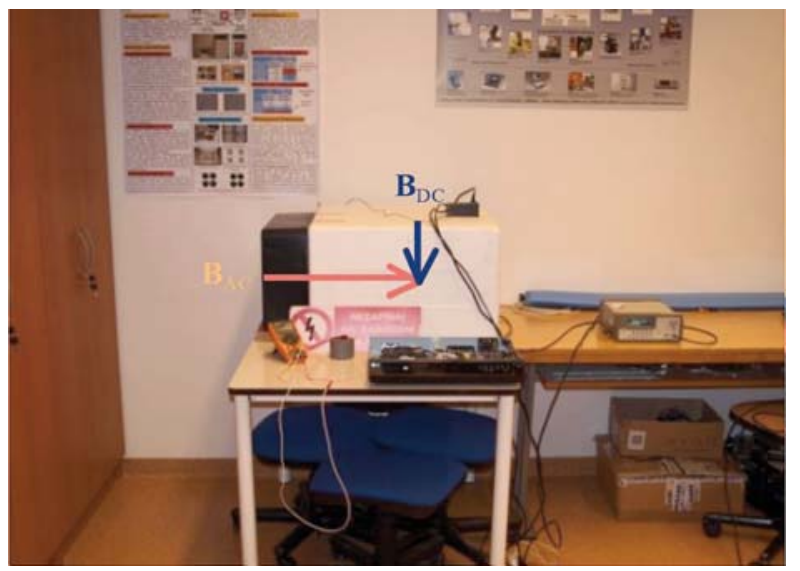

Fig. 7 Perpendicular combination of the applied EMFs parallel to the B vector of DC field represented by the Earth's magnetic field (measured as $47 \mu \mathrm{T}$ ). The second experiment was focused on the perpendicular combination of the applied electromagnetic fields. The whole incubator was placed on its side to the vertical plane, which is demonstrated in Fig. 7. Then another 66 hour lasting exposure started under the same conditions as in the previous case.

Comparison of results portrays an interesting picture, which is shown in Fig. 8. The ratio in the figures is given by the growth area ratio between the cell colonies of exposed and unexposed samples.

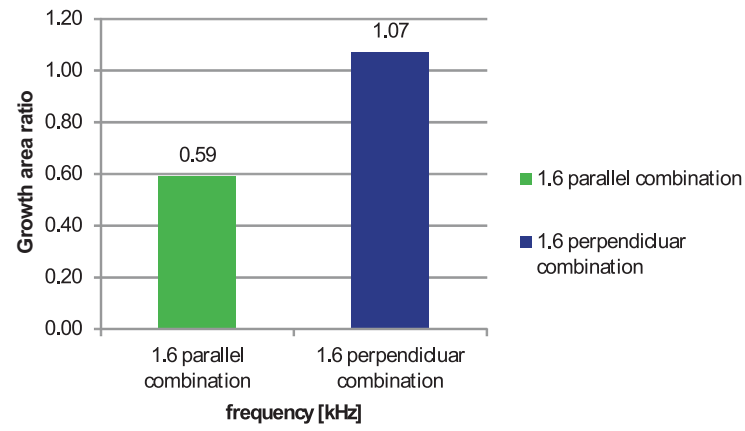

Fig. 8 Comparison between the influence of parallel and perpendicular combination of LF EMFS

For a better reliability each of the experiments is repeated under the same exposure conditions. According to Student's t-test, the obtained results are statistically comparative to the previous experiments, as it is demonstrated in Table 1 .

Example of Student's t-test for first batch of experiments Table 1 t-Test: Paired Two Sample for Means $\quad \alpha \quad 0.05$

\begin{tabular}{lrr}
\hline & \multicolumn{1}{c}{ cont } & exp \\
\hline mean & 28565.56 & 17037.67 \\
Observations & 9 & 9 \\
Pearson Correlation & 0.915305 \\
Hypothesized Mean Difference & \multicolumn{2}{c}{0} \\
df & 8 \\
t Stat & 9.500 \\
P(T<=t) one-tail & 0.000 \\
T Critical one-tail & 1.860 \\
P(T<=t) two-tail & 0.000 \\
T Critical Two-tail & 2.306 \\
\hline
\end{tabular}

\section{Conclusion}

The presented article summarizes recent theories about the LF EMF influence to the biological structures. As a confirmation of previous experiments aligned with the presented theories, the 
new coil system was designed to achieve better homogeneity of the applied LF EMF. The new exposure system was used for the experiments focused on the biological effect of parallel and perpendicular combination of applied time-varying and static magnetic field.

The results from the presented experiments gave ground for the following conclusions. First, a biological effect was clearly observed at given frequency that confirms findings from $[12,13]$.

Second and probably foremost, the inhibitive proliferative response of the biological samples was observed during the application of parallel combination of AC and DC electromagnetic field, while the perpendicular combination showed no effect at given frequency. This finding could support mainly the Lednev's IPR theory about the influence of superposed AC and DC magnetic fields to the bound ions on the cell membrane surface. On the other hand, the anti-proliferative effect could be also explained by the Frohlich's theory. For further work it would be interesting to investigate the influence in wide frequency range of applied electromagnetic field. Especially the sub-harmonic frequencies of the base frequency presented in this work could provide considerable results especially verification of the IPR model predictions seems to be very interesting.

From afore mentioned it seems that both of the compared mechanisms could be used for explanation of the observed yeast behavior. But there are still some uncertainties, so the authors assume that the correct interpretation of the presented results could be achieved by synthesis of the described models. In advance, the presented findings give a reason to believe that such behavior could be exploited with advantage in therapeutic application targeting specific cell/tissue types.

\section{References}

[1] CONE Jr., C. D.: Unified Theory on the Basic Mechanism of Normal Mitotic Control and Oncogenesis. J. of Theoretical Biology, vol. 30, pp. 151-181, 1971.

[2] BARABAS, J., RADIL, R.: Investigation of Cellular Transmembrane Potential Variations via Exogenous Low Frequency Electromagnetic Fields. Clinician and Technology J., vol. 40, pp. 41-45, 2010.

[3] WEBB, S. J., STONEHAM, M. E., FROHLICH, H.: Evidence for Non-thermal Excitation of Energy Levels in Active Biological Systems, Physics Letters A, vol. 63, pp. 407, 1977.

[4] POKORNY, J.: Cancer Physics: Diagnostics Based on Damped Cellular Vibrations in Microtubules. Eur Biophys J., vol. 40, pp. 747-759, 2011.

[5] CIFRA M., POKORNY, J., JELINEK, F., HASEK, J.: Measurement of Yeast Cell Electrical Oscillations around 1 kHz. PIERS Proceedings. Cambridge, USA, pp. 780-784, 2008.

[6] LEDNEV, V. V.: Possible Mechanism for the Influence of Weak Magnetic Fields on Biological Systems. Bioelectromagnetics. vol. 12, No. 2, pp. 71-75, 1991.

[7] LIBOFF, A. R.: Cyclotron Resonance in Membrane Transport. Interactions between Electromagnetic Fields and Cells. Plenum Press, pp. 287-296, 1985.

[8] ADAIR, R. K.: Criticism of Lednev's Mechanism for the Influence of Weak Magnetic Fields on Biological Systems. Bioelectromagnetics, vol. 13, pp. 231-235, 1992.

[9] ENGSTROM, S.: Dynamic Properties of Lednev's Parametric Resonance Mechanism. Bioelectromagnetics, pp. 58-70, 1996

[10] LEDNEV, V. V.: Article in Russian, Biofizika, vol. 41, p. 815, 1996.

[11] BARABAS, J.: Proliferative Response of Two Different Saccharomyces Cerevisiae Strands to Extremely Low Frequency Electromagnetic Fields. POSTER 2011: $15^{\text {th }}$ Intern. Student Conference on Electrical Engineering, 2011, Prague, ISBN/ ISSN978-80-01-04806-1.

[12] CAP, I., BARABAS, J.: Low Frequency Electromagnetic Fields: Friend or Foe? Communications - Scientific Letters of the University of Zilina, vol. 1, pp. 13-17, 2011.

[13] BARABAS, J., CAP, I.: S. Cerevisiae Growth Regulation via Exogenous Low Frequency Electromagnetic Fields. Proceedings Trends in Biomedical Engineering, pp. 141-144, 2011. 